\title{
Método ArQueológico EduCATivo
}

\author{
EDUCATIONAL ARCHAELOGICAL METHOD
}

\section{LAURA Helou - Romina MAgAllanes - CAROLINA MONZÓN - JORGELINA RUSSO \\ Colegio Joan Miró- Universidad Nacional de Rosario laurahelou_1@hotmail.com -}

romina_magallanes@yahoo.com.ar-caromonzon06@hotmail.com -jorgelinarusso@colegiojoanmiro.edu.ar

RECIBIDO: 28 DE AGOSTO DE 2018

ACEPTADO: 20 DE OCTUBRE DE 2018

Resumen: El siguiente trabajo expondrá un Método denominado Arqueológico Educativo, que nuestro grupo de Estudios Filosóficos viene desarrollando en los últimos años.

Para esto, realizaremos una exposición que recorre nuestras fuentes conceptuales conformadas por autores como Heidegger, Foucault, Deleuze y Guattari, y también pensadores vinculados a la Práctica de la Filosofía con niños específicamente.

Nuestro propósito es presentar la construcción de una Práctica Educativa Filosófica como aquella que apuesta a abordar los saberes escolares tradicionales - de los Programas de los Ministerios de Educación en la Argentinadeconstruyendo, desmontando, descomponiendo sus áreas curriculares a partir de las propuestas de los autores mencionados y de la Metodología del Programa de Filosofía Infantil, proyectado por Lipman, complejizado y problematizado por diversos pensadores, como también agenciado por una apropiación que nuestra Institución Educativa se permitió realizar.

Así, anhelamos que la Práctica Filosófica ingrese en el Sistema Educativo para repensar y conmocionar sus cimientos.

Palabras claves: método, arqueología, educación, infancia, filosofía infantil.

Abstract: The following paper will explain a Method called Educational Archeology. That one has been developed by our Philosophical Group in recent years. 
In order to accomplish this, we will make an exposition that goes through our conceptual sources conformed by authors like Heidegger, Foucault, Deleuze and Guattari, and also thinkers linked to the Practice of Philosophy with children specifically.

Our purpose is to present the construction of a Philosophical Educational Practice as one that aims to approach traditional school knowledge -of the Programs of the Ministries of Education in Argentina- by deconstructing, decomposing their curricular areas based on the proposals of the authors mentioned and the Methodology of the Children's Philosophy Program, projected by Lipman, complex and problematized by different thinkers, as well as agency by an appropriation that our Educational Institution was allowedto perform.

Thus, we hope that Philosophical Practice enters the Educational System to rethink and shake up its foundations.

Keywords: method; archeology, education, infancy, children’s philosophy.

A todo hombre le llega el momento en que debe pronunciar este "yo puedo" que no refiere a ningunacerteza ni a ninguna capacidad específica, y que sin embargo lo empeña y lo pone completamente en juego. Este "yopuedo" más allá de toda facultad y de todo saber hacer, estaafirmación que no significa nada, pone al sujeto inmediatamente frente a la experiencia quizás más exigente $-\mathrm{y}$ no obstante ineludiblecon que le es dado medirse: la experiencia de la potencia.

Giorgio Agamben, La potencia del pensamiento.

¿Existe un método educativo que pueda implementarse en las escuelas sin ser una problematización tanto de los currículos obligatorios por los Ministerios correspondientes como de la propia práctica docente? ¿O se trata tal vez de "inventar" a partir de un camino (methodós) propio, dialogado que cree que la práctica filosófica infantil puede posibilitar e intensificar una experiencia de

Ver, Kohan, Walter: "El maestro inventor. Simón Rodriguez", Caracas, Ediciones del Solar, 2016. También, Rodriguez, Allan, Berle, Simone, Kohan, Walter, "Filosofía e Filosofía e Educaçãoemerrância: inventar escola, infâncias do pensar", NEFI, Río de Janeiro, 2018, pp. 9-17. 
escuela a partir de preguntas, de lecturas, de investigación y de pensar las propias vivencias y deseos potentes?

A continuación expondremos el Método que estamos elaborando a partir de la aplicación del Programa de Filosofía Infantil a las áreas curriculares escolares del nivel primario.

Este es un camino de casi dos décadas, en donde comenzamos con la implementación del Programa de Filosofía con Niños, para pasar luego a la instancia de generación de nuestra propia práctica de Filosofía Infantil, continuando con una experiencia llamada Pensar los Saberes que es la que comienza a dar lugar, en este último tiempo, al Método que dimos en llamar Arqueológico Educativo, ya en el marco de una formalidad constructiva metodológica, vivencia que expondremos seguidamente.

Definimos nuestroMétodo Arqueológico Educativo como aquel que apuesta a abordar el saber escolartradicional -reflejado en los Programas organizados por los Ministerios de Educación en la Argentina-deconstruyendo, ${ }^{2}$ es decir, desmontando y analizando

${ }^{2}$ Tomamos el término "deconstrucción" siguiendo los bordes de los conceptos de Destruktion de Heidegger y la relectura del mismo que lleva a cabo Derrida. Como indica Gragnolini: "Heidegger, por su parte, denomina "onto-teología" a esa historia de la metafísica en la que, cada vez que es planteada la pregunta por el ser, se responde a la misma con un "ente supremo" (Theós), y su método de "destrucción de la historia de la metafísica" se une al "paso atrás" para buscar el origen de ese olvido, que confunde el ser con el ente. (...) El deconstruccionismo se presenta, combinando algunas de estas formas, como un habitar las estructuras de la metafísica para mostrar las fisuras de las mismas (...) un ejercicio del pensar que supone, más que intentar "fugarse" de la metafísica, permanecer en ella, realizando un trabajo que implique horadarla desde sus mismas estructuras. Algo que Nietzsche, con la figura del filósofo topo, ya se había propuesto. La tarea nietzscheana consiste en el análisis de la cultura, de sus presupuestos y fundamentos, con el objeto de llevar hasta el estallido ciertos conceptos y términos que rigen el pensar de occidente".

"El deconstruccionismo es un modo de habitar las estructuras metafísicas para llevarlas hasta su límite: solicitación (en el sentido etimológico de "hacer temblar") que permitirá que dichas estructuras muestren sus "fisuras". 
sus distintas dimensiones -la de la Matemática, de las Ciencias Naturales y Sociales, de la Lengua, de la Ética, y demás áreas curriculares-, desde la Metodología de la investigación del Programa de Filosofía Infantil, promovido y sistematizado por M. Lipman y A. Sharp, problematizado por diversos pensadores,como también originado en una inspiración teórica-práctica propia que nuestra Institución Educativa, el Colegio Joan Miró, se permitió realizar.

\section{Breve recorrido de un camino que se rizomatiza}

Como indica Barrientos "El germen de la Filosofía para Niños se encuentra en un filósofo estadounidense que fue alumno del pragmatista John Dewey" 3. Que el pragmatismo lógico recorre toda la obra de Lipman es coherente con su formación y atención metodológica sobre el razonamiento lógico y una intención de formación de sus estudiantes en una democracia racional y liberal. ${ }^{4}$

Cragnolini, Mónica: "Derrida: deconstrucción y pensar en las "fisuras", en Ciclo "El pensamiento francés contemporáneo, su impronta en el siglo, Conferencia en la Alianza Francesa, Buenos Aires, 30 de setiembre de 1999.

Esta "dislocación" como la denomina Gragnolini da lugar a una desedimentación del valor de conceptos fundamentales, en nuestro caso, de la formación educativa primaria, saturados de seguridad incuestionable tanto en los contenidos curriculares obligatorios como en su abordaje didáctico. En este punto, aunamos dichas perspectivas con la de Michel Foucault en su arqueología del saber.

${ }^{3}$ Barrientos, José, "La experiencialidad como respuesta a la tendencia analítica de la filosofía para niños" en Childhood\&phylosophy, V. 12, n. 25, Río de Janeiro, st- dez. 2016, pp. 519-542.

${ }^{4}$ En Argentina, la teorización y práctica de El PFpN de Lipman funciona en el Centro de Investigaciones en el Programa Internacional Filosofía para Niños (C.I.Fi.N) presidido por Stella Accorinti, que retomar y repite las propuestas lipmanianas. 
Muchos teóricos se han apoyado en esta única perspectiva para abordar el trabajo que vincula de modo siempre inquietante el filosofar con la infancia. Así,

se han fundado en muchos autores (como Oscar Brenifier) en patrones analíticos y conceptuales. Aún cuando han estudiado elementos afectivos y emocionales, las metodologías desarrolladas en el aula responden a este formato, es decir, la principales herramientas de trabajo acostumbran a ser la argumentación, la creación de definiciones, el diseño de descripciones, el análisis conceptual, la confrontación de tesis o la construcción de nuevas teorías o estructuras ideacionales, entre otras. Esto es debido al tipo de racionalidad sobre la que descansan muchas teorías de Filosofía para Niños: una filosofía entendida como proceso argumentativo que establece y critica hipótesis y estructura analíticamente la realidad. Sin embargo, el siglo XX y XXI ha traído otras formas de filosofía: la razón vital y narrativa de Ortega y Gasset, la razón poética de María Zambrano, la razón dramática de Sartre, los sones místicos de Heidegger y la razón ontológico-estética de Gadamer o de Benjamin, por poner sólo algunos ejemplos. Algunos autores han realizado aproximaciones estéticas en Filosofía para Niños, por ejemplo, las reflexiones artísticas de Kohan y Vaksman han trabajado con objetos de arte y Sátiro ha materializado traducciones desde lo plástico a lo oral y viceversa. No obstante, es urgente reflexionar sobre una modalidad de Filosofía para Niños cuyo foco de interés no sea exclusivamente la reflexión sobre nuevo material sino el trabajo con material nuevo. De esta forma, se crearía un pensamiento más allá de los modos analíticos tradicionales: comprender no consistiría sólo en una acción crítica sino en una forma de pensar sin palabras. El presente trabajo propone una aproximación a la Filosofía para Niños desde una de esas racionalidades: la experiencia de la vida o experiencialidad. ${ }^{5}$

Atendiendo tanto a la historia del Programa de Filosofía "para"
Niños, "con" niños, pasando por diversos pensadores que abren e

${ }^{5}$ Barrientos, José, "La experiencialidad como respuesta a la tendencia analítica de la filosofía para niños" en Childhood\&phylosophy, V. 12, n. 25, Río de Janeiro, st- dez. 2016, pp. 519-542. 
intensifican el panorama de la Filosofía y la Infancia y su práctica hacia otras obras que actualizan e incluyen trabajos de filósofos como Heidegger, Deleuze, Guattari, Foucault, Agamben, dejados de lado por las lecturas repetitivas del Programa inicial de Lipman, como también los abordajes de pensadores latinoamericanos integrando textos y problemáticas propios de nuestra cultura, ya no sólo ligados a la lógica sino también a lo político, lo estético, lo existencial en general, y al modo fundamental del preguntar, es que nos proponemos reunir y discutir puntos de vista diversos sobre nuestra práctica para enriquecerla eintensificarla. Siguiendo a Kohan en este sentido:

Existe, por cierto, un saber específico de la práctica filosófica, un uso de los conceptos, un modo de argumentar, una historia de la filosofía. Pero antes que eso, es necesario preguntarse. No se trata de saber hacer preguntas, sino de hacerse preguntas uno mismo. Vivir uno mismo las preguntas. Ser esas preguntas. Sin esa puesta en cuestión de sí mimo, de su saber, de su relación con los otros y con la realidad que lo rodea, difícilmente sea fructífera la puesta en cuestión de lo demás. La pregunta filosófica no es solamente la búsqueda de la respuesta, es también planteo de un problema, es apertura de un camino. En este sentido, es diferente de otros tipos de preguntas que suelen tener lugar en la escuela, preguntas para saber qué sabe el alumno, preguntas para averiguar la solución. La pregunta en filosofía, y en filosofía con niños también, inaugura una indagación. Es la indagación de quien no pregunta para saber más sino para abrir los saberes que circulan en la escuela. Se pregunta para dejar de saber un poco lo mucho que creemos saber, para revisar los saberes que nos constituyen en lo que somos y que no nos dejan ser otra cosa. Pero las preguntas no surgen solas, o al menos, no en todos los casos, no siempre. He aquí, entonces, un sentido de la práctica filosófica: sacar a la luz las preguntas, sacar a la luz que admite tanto la idea de descubrimiento como la de creación: las preguntas pueden estar detrás de los supuestos, del sentido común, o pueden no estar y surgen allí donde antes no había nada. ${ }^{6}$

\footnotetext{
${ }^{6}$ Kohan, Walter, Waksman, Vera: Filosofía con niños. Aportes para el trabajo en clase, Novedades educativas. Buenos Aires, 2005, pp. 95-6.
} 
Por esto, consideramos que, con dicha apuesta, la Práctica Filosófica ingresa intensa y formalmente en el Sistema Educativo para volver a pensar $\mathrm{y}$, por qué no, para conmocionar sus cimientos. La incorporación de un permanente procedimiento de deconstrucción de las mencionadas dimensiones de los saberes aspira a develar sus condiciones de producción social, histórica, política y cultural.

\section{Perspectivas del método}

Por otra parte, el aporte de este Método nace y se proyecta en una perspectiva ética abierta por Foucault: es decir, una ética como relación entre uno con uno mismo y con los otros seres, siempre en vinculación con los saberes que nos rodean y nos constituyen subjetivamente y que, simultáneamente, constituimos. ${ }^{7}$

Para el Método Arqueológico Educativo, es esencial exaltar esta visióny potenciar un movimiento educativo que resalte no a un alumno-infante pasivo y vacío que recibe contenidos de un docente- adulto activo y poseedor de un saber definitivo y acrítico, - conceptos que, desde hace décadas, se intentan erradicar - sino a sujetos dialógicos que, a través de Comunidades de Investigación dando, entonces, un paso más, se impliquen a sí mismos en sus devenires sujetos, mediante creaciones, críticas, cuidado de sí y de los otros, donde los saberes, además de ser discursos a conocer, son experiencias a habitar, contenidos a contextualizar e historizar, y modos de transitar la infancia -tanto los alumnos como los docentes-:la infancia como potencia, como prácticas de apertura, posibilidades de ser, crear, vivir. ${ }^{8}$

\footnotetext{
${ }^{7}$ Foucault, Michel: La hermenéutica del sujeto. FCE, Buenos Aires, 2006.

${ }^{8}$ Gagnebin, Jeanne Marie: "Infância e pensamento" ", en Sete Aulas sobre Linguagem, Memória e História. Imago, Río de Janeiro, 2012, pp. 168-178:
} 
señala que la noción de infancia es profundamente histórica, y que la vinculación entre la misma y la filosofía es tan antigua como la filosofía misma. Presenta dos líneas: una que nace con Platón, atraviesa la pedagogía cristiana de San Agustín y llega hasta el racionalismo cartesiano. Esta línea piensa a la infancia como un "mal necesario, una condición próxima al estado animalesco y primitivo", los niños son seres privados de razón, deben ser corregidas sus tendencias salvajes, irreflexivas, egoístas, que amenazan una construcción social consensual.También Freud es incluido en esta línea al considerar la necesidad de la represión para llegar a "la sublimación creadora de valores culturales". La otra línea, también nace con Platón, atraviesa el renacimiento con Montaigne y llega a Rousseau. Esta línea asegura que no sirven de nada las reglas, normas, contenidos con los que se pretende llenar a los niños, sino que la educación verdadera consiste en la preparación adecuada de sus almas para que ellas por impulso propio y natural puedan crecer y desenvolver la inteligencia de cada niño según sus tiempos e intereses propios. Gagnebin resalta que la palabra infancia no se refiere a una determinada edad, sino más bien a la ausencia de discurso. La autora se pregunta cuál es el significado de esa ausencia primordial.Para Rousseau esa ausencia, el no hablar esconde nuestra proximidad con el animal y nuestro alejamiento de la simplicidad de su instinto. Para San Agustín, la infancia reúne lo salvaje del animal y la disponibilidad del hombre para el mal. La infancia es el testimonio del pecado marcado en nosotros, contra el que solo podemos luchar cuando salimos de ella. La infancia es asimismo "un lugar de perdición y confusión" para el Renacimiento y el racionalismo. Descartes, por ejemplo, ve en ella el lugar de error, del "preconcepto, de creencia ciega", vicios de los cuales el pensamiento debe liberarse. Si deja de ser espacio del pecado, sin embargo continúa considerándose como la permanencia en la "sin-razón". Como Platón, Descartes plantea una reforma de la educación, ya que la educación tradicional no percibe el "brotar de una razón balbuciente que, muchas veces es sofocado por un cúmulo de informaciones oscuras y paradojales". Debemos librarnos de la mejor manera de ese período infeliz que es la infancia, creando otras condiciones, que propicien el crecimiento rápido de la luz natural del alma, del nous platónico y de la razón cartesiana para volvernos adultos, o como decía Kant salir de la minoridad. El Iluminismo celebra junto a la edad de la razón que es la edad adulta, la emancipación ética y política que es imposible en la edad de la sin- razón que es la infancia. .En oposición a la razón calculista, Rousseau valoriza una naturaleza primera que lo conduce a su teoría de la deformación, de la decadencia que tiene lugar a través de la historia y la cultura. Ya no se trata de corregir la naturaleza infantil sino escuchar esa voz de la naturaleza del infante, ayudar a su desenvolvimiento armonioso, no a las dictadas por las convenciones 
De este modo, hablamos de Métodono en el sentido sistemático de un esquema, sino siguiendo su etimología, comometa yhodós: "a través de un camino". Dicho Método es Arqueológico, porque se propone desmontar y deconstruir los saberes en sus condiciones de producción y origen, y mostrar cómo dichos saberes no son conocimientos cerrados ni atemporales sino que nacen de relaciones de poder, de situaciones donde los ámbitos sociales, políticos y económicos, entre otros, se ponen en juego. ${ }^{9} \mathrm{Y}$, finalmente, es Educativo porque se despliega en un recorrido centrado en el espacio escolar, lugar justo para pensar éticamente lo real actual, nuestro habitar en él, y a nosotros mismos.

Así, este Método implica en su práctica incipiente la utilización de las herramientas filosóficaso movimientos básicos propios del Programa de Filosofía Infantil más la incorporación de otras afines y que construimos en consonancia al mismo. Estas son la "historización", "contextualización", "relativización", "escepticización", “desmanualización”, "rememoración”, entre otras.

Con "desmanualización" nos referimos a desnaturalizar la utilización de manuales como textos absolutos, revisar y contrastar el tratamiento de una temática desde diferentes enfoques $\mathrm{y}$

sociales sino las más cercanas a la naturaleza de las facultades infantiles, más auténticas e inocentes opuestas al mundo adulto que las pervierte. La infancia aparece como un paraíso perdido.

Sin embargo, la contemporaneidad ha expuesto otros modos de ver la infancia. Según la autora, los trabajos de W. Benjamin, J. F. Lyotard y G. Agamben dan nuevas pistas sobre la relación de la infancia y el pensamiento. Así, Agamben, Giorgio: Infancia e historia. Destrucción de la experiencia y origen de la historia. Adriana Hidalgo. Buenos Aires, 2007, p. 64: "Una experiencia originaria, lejos de ser algo subjetivo, no podría ser entonces sino aquello que en el hombre está antes del sujeto, es decir, antes del lenguaje: una experiencia "muda" en el sentido literal del término, una in-fancia del hombre cuyo límite justamente el lenguaje debería señalar".

${ }^{9}$ Foucault, Michel: La arqueología del saber, Siglo XXI, Buenos Aires, 2002. 
desmontar sus estrategias de producción.

La "historización" consiste en situar cada "contenido conceptual" como acontecimiento temporal, no presentarlo como una tema neutro y aséptico frente a los sucesos en medio de los cuales esos temas tuvieron lugar. Tanto en su momento de formación como en el presente, problemática desde la cual también lo analizamos. Aquí hacemos hincapié en la noción de "rememoración" heideggeriana, que amplia y profundiza la historización, ya que visibiliza cada problemática a abordar no desde una mirada representativa y explicativa, sino dando un paso más hacia atrás, atendiendo a cómo tuvo lugar nuestro habitar entre esas problemáticas, no convertidas en objetos a reducir a un razonamiento sino como textos que nos ligan y que hemos construido, y que nos construyeron como existentes, textos en los que habitamos y debemos vigilar en el modo de la pregunta . Así, a partir de la reunión de estas herramientas, estos movimientos del pensar, es como concebimos el "contextualizar", es decir, conducirnos junto a un contenido a su momento y espacio de formación, sus y nuestras modificaciones respecto de él. Tanto la "relativización" como la "escepticización" son pensadas como actitudes en el abordaje de los textos, de la información, de la circulación de los mismos; una actitud de cautela y de duda, que acompañan a todo el Método en cada una de sus instancias. Así, junto a todas las herramientas, se busca la desnaturalización de la convenciones que están compuestas por "sentidos comunes" y presupuestos no pensados fundamentalmente frente a todo lo instituido. ${ }^{10}$

\footnotetext{
${ }^{10}$ Durante todo el desarrollo de trabajo del Método Arqueológico Educativo, del cual aquí se mostrarán algunos breves ejemplos, éste es atravesado y forjado por y en las herramientas filosóficas o movimientos básicos indicados. En los ejemplos que se mostrarán más adelante, al tratarse de actividades realizadas con estudiantes de Primer Grado, las herramientas-movimientos utilizados son las creadas por el Método, ya indicadas, y también las propuestas desde Lipman y
} 


\section{Modelo epistemológico}

Asimismo, hacemos hincapié en la índole de imbricación rizomática de los saberes escolares mencionados, entendiendo por esto una dilatación de los denominados "contenidos conceptuales"un modo de denominar la trasmisión de saberes que ha llevado a cabo la estructura educativa inicial, primaria, media y superior en nuestro país-que comienzan a ser tratados como "rizomas".

Pensar las problemáticas educativas como rizomas implica, como muchos de ustedes saben, entenderlos como un modelo epistemológico, planteado por Deleuze y Guattari, donde la organización de los elementos no sigue líneas de subordinación jerárquica con una raíz dando origen a múltiples ramas, sino que cualquier elemento puede afectar o incidir en cualquier otro. En el modelo del árbol jerárquico tradicional de organización del conocimiento - como las taxonomías y clasificaciones generalmente empleadas por las ciencias- lo que se afirma de los elementos de mayor nivel es verdadero en los elementos subordinados, y no a la inversa. En un modelo rizomático, en cambio, cualquier afirmación sobre un elemento puede incidir en la concepción de otros elementos de la estructura, sin importar su posición recíproca. El rizoma, así, carece de centro.

De este modo, pensando los saberes curriculares desde la perspectiva rizomática, éstos se desmontan, se deconstruyen y se abren a la construcción plural de la Comunidad Educativa. Por lo tanto, parte de los llamados "contenidos procedimentales" y

Sharp hasta nuestros días en trabajos de diferentes pensadores como G. Santiago, O. Brenifier, entre otros, pero haremos énfasis en las siguientes: preguntar, hipotetizar, definir,analizar, dar ejemplos y contraejemplos, realizar analogías, escuchar, practicar la empatía. Sabiendo, por otra parte, que son movimientos que se cruzan y se dan lugar unas a otras en forma constante.

${ }^{11}$ Deleuze, Gilles; Guatari, Félix: "Rizoma", en Mil Mesetas. Capitalismo y esquizofrenia, Pre-textos, Valencia, 2004, pp. 9-30. 
"contenidos actitudinales" -otras de las categorías estipuladas por los formatos ministeriales- se hallan contemplados en el modo del rizoma. Es decir ni partimentados, ni jerarquizados, sino dinamizados e imbricados con las denominadas "herramientas filosóficas" correspondientes a los tres tipos de pensamiento planteados, desdeLipman y, en adelante, otros autores, como son el pensamiento Crítico, Creativo y Ético, ${ }^{12}$ y a nuestros propios aportes institucionales; lo que hace delos mismos un espectro que los incluye y que conduce a unaco-constitución fundante.

El rizoma se abre, además, en otros puntos de fuga de importancia fundamentalpara nosotros que reponen lo que creemos insolvencias como son los tratamientos curriculares de los "textos" y la ausencia de preguntas.El texto normalizado en géneros inmóviles, con partes constitutivas y distintivas inermes, como por ejemplo, la confinación de la poesía al uso de la rima desestima la riqueza y la significación filosófica que un texto conlleva sobre sí y, a su vez, habilita a posibilidades nunca clausuradas de lecturas y preguntas.

${ }^{12}$ Cázares Aponte, Leslie: Imbricar Filosofía para Niños y Jóvenes en los centros escolares. Una perspectiva de inclusión escolar para toda la comunidad educativa, Octaedro Recursos, Barcelona, 2014, p. 20 suma el pensamiento colaborativo: "En este libro hablaré del pensamiento colaborativo, que si bien está inserto en los tres mencionados, el hecho de declararlo y enunciarlo de manera aislada permitirá al docente frente al grupo planificarlo y procurarlo de manera más explícita. Este cuarto nivel de pensamiento se ha trabajado transversalmente en toda la metodología a través de la propuesta de las comunidades de indagación. La idea de resaltar esta acción colaborativa como un cuarto nivel de pensamiento contempla la posibilidad de identificar desempeños y competencias educativas propias para estimularlas en paralelo al pensamiento crítico, ubicándolas en un contexto con el pensamiento creativo para dimensionar la creación social y con el pensamiento cuidadoso para enfatizar los valores de cooperación entre sus pequeñas y grandes comunidades de aprendizaje". Luego, lo que consideramos más significativo: "Reconocer a cada uno de los miembros del grupo como seres con historia, presente y futuro de acuerdo a un contexto, es dimensionar a las personas que nos acompañan en el proceso de pensar como sujetos integrales de los cuales es posible aprender" p. 80 . 
Como dice Maximiliano López:

Texto es todo aquello que se nos presenta como un signo. Desde ese punto de vista, los textos que pueden ser utilizados en una experiencia de "filosofía con niños" son innumerables: filmes, pinturas, objetos, situaciones, poemas, narraciones, textos académicos, leyendas populares. Todo puede despertar el pensamiento. Estrictamente hablando, todo el mundo y cada una de sus partes pueden ser considerados un signo, dado que signo es todo aquello que consideramos como teniendo algo para decir, como teniendo un sentido. ${ }^{13}$

Siguiendo este matiz de la noción de texto nos interesa acercarlo a la noción de "cosa", en el sentido que la piensaHeidegger, es decir, como un espacio que coliga a los humanos en su habitar en el mundo:

¿Cuándo y cómo llegan las cosas como cosas? No llegan por las maquinaciones del hombre. Pero tampoco llegan sin la vigilancia atenta de los mortales. El primer paso hacia esta vigilancia atenta es el paso hacia atrás, saliendo del pensamiento que sólo representa, es decir, explica, y yendo hacia el pensamiento que rememora. ${ }^{14}$

Así, intentamos adentrarnos mediante nuestro Método Arqueológico en una práctica compleja que consiste en considerar texto-cosa-pregunta-lectura-práctica ${ }^{15}$ como un rizoma que por

13 López, Maximiliano: Filosofía con niños y jóvenes. La comunidad de indagación a partir de los conceptos de acontecimiento y experiencia trágica, Noveduc, Buenos Aires, 2008, p. 98.

${ }^{14}$ Heidegger, Martín: "La cosa", en Conferencias y artículos, Ediciones del Serbal, Barcelona. 1994, p. 12.

${ }^{15}$ Como un movimiento rizomático complejo antes que como la presentación asertiva estática dividida en tema/actividades/explicación del docente/evaluación, y poco dialógica de un contenido a trabajar docentes y alumnos. 
medio de la vivencia de la "clase" como experiencia de Proyectosrizomáticos, ${ }^{16}$ devele la índole arqueológica, es decir, susceptible de descomponer en las partes que constituyeron genealógicamente cada unidad de saber escolar; como también rememorativa en el sentido heideggeriano: dando un paso hacia atrás, vigilando cómo aconteció nuestro habitar entre cosas, ya no convertidas en objetos a explicar por medio de la representación, sino como textos que nos ligan y que hemos construido, en los que habitamos y debemos vigilar en el modo de la pregunta .

De este modo, podremos atravesar y movilizar, en una forma que focalice en la contextualización, la historización, la desmanualización, con una actitud escéptica, crítica, creativa, ética y rememorativa las problemáticas que el currículo plantea. En algunos casos cuestionándolas, en otros ampliándolas y siempre interrogándolas.

Una de las prácticas fundamentales que el Método Arqueológico está llevando adelante es que cada grado de la Educación Primaria, se trace anualmente diversos Proyectos vinculados por la problemática texto-cosa-pregunta-lectura-práctica, para comenzar a desplegar, construir y reconstruir sus dimensiones rizomáticas.

\footnotetext{
${ }^{16}$ Para profundizar la idea de Proyectos rizomáticos ver la nota anterior. Se trata de "tomar" una "cosa" rememorar su co-constitución histórica con actitud escéptica y relativa, estudiarla desde las informaciones disponibles sobre ella y hacerla habitar sus y nuestros tiempos, desde las áreas curriculares escolares: Lengua, Ciencias Naturales, Ciencias Sociales, Matemática, Construcción Ética y Ciudadana, Tecnología, y Áreas de Especialidades. Mostrando panoramas móviles de los saberes y de las "cosas": temas, textos, acontecimientos que los constituyeron; y a la vez cómo se fue dando cuenta de ellos. El proyecto busca imbricar a partir de una "cosa", texto, palabra, evento, los diferentes ámbitos que le dieron lugar sin aislar dicho tema en compartimentos estancos, que así también estanca la dinámica intricada y compleja de los saberes.
} 


\section{Ejemplo de aplicación práctica}

Lo que vemos aquí es un ejemplo, entre muchos otros que hacen a la conformación de nuestro Método de los Proyectos en Primer grado. Se parte del Rizoma "fósil", permitiendo deconstruir dicho texto a partir del abordaje desde los distintos espacios curriculares articulando los conocimientos propios de cada uno, en este primer caso en el campo disciplinar de la Lengua, y como desarrollaremos más adelante en las áreas deCiencias Sociales, Ciencias Naturales, Matemática, Construcción ${ }^{17}$ Ética y Ciudadanay Tecnología.

\footnotetext{
${ }^{17}$ Modificamos el término "Formación", que es el correspondiente al nombre oficial del Área EducativaFormación Ética y Ciudadana, por el de "Construcción", que consideramosen varios sentidos: por un lado, como un "liberar (...) la conversación con lo sido" (Heidegger, Martín. ¿Qué es eso de filosofía? Sur. Buenos Aires. 1960, p.19), entendiendo por "lo sido" como aquello que "sigue sin cesar vivo y activo" (Carpio, A. traductor de ¿Qué es eso de filosofía? Sur. Buenos Aires. 1960, p. 199), no con un pasado que nada tiene para decir ni un presente que no puede dialogar con lo que aún está latente, sino una actitud que desmonta aquello que permanece de otras formas y que al hacerlo crea un diálogo arqueológico con aquello "sido"; por otro lado, como lo opuesto a "formar" que implica un movimiento de cierta imposición de una forma a algo informe, la manipulación de un estudiante vacío y neutro por parte de un o una docente llena de contenido a verter y a moldear. Por ello, construir se vincula con el preguntar, el preguntar despierta no impone, como sigue diciendoHeidegger la pregunta se despierta "cada vez que se ha oscurecido y enredado aquello por cuya esencia se pregunta, cuando al tiempo se ha vuelto vacilante, o aún se ha quebrajado, la relación del hombre con lo preguntado" (Heidegger, Martín. ¿Qué es eso de filosofía? Sur. Buenos Aires. 1960, p. 24), por esto, tanto el presente como el pasado construyen lo sido desde la pregunta que es convocada por presente que desmonta. Por último, la Construcción Ética y Ciudadana solo puede tener lugar como un saber en movimiento activo que interroga lo ético y crea convivencias en acuerdos dialógicos y no como una doctrina a transmitir que repite contenidos y normas externas al presente convocado y convocante.
} 


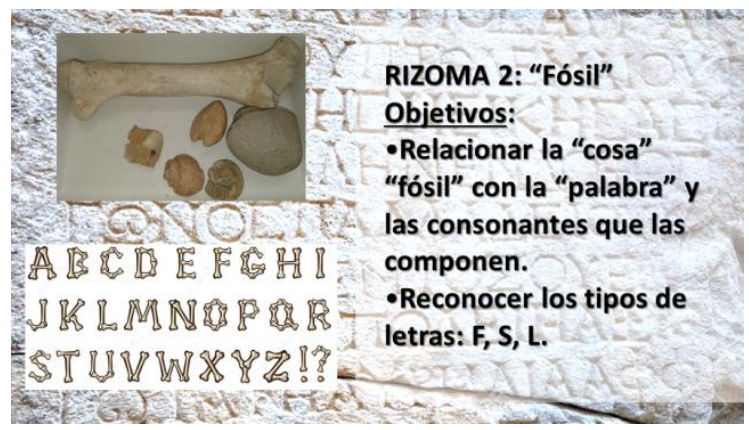

Figura 1

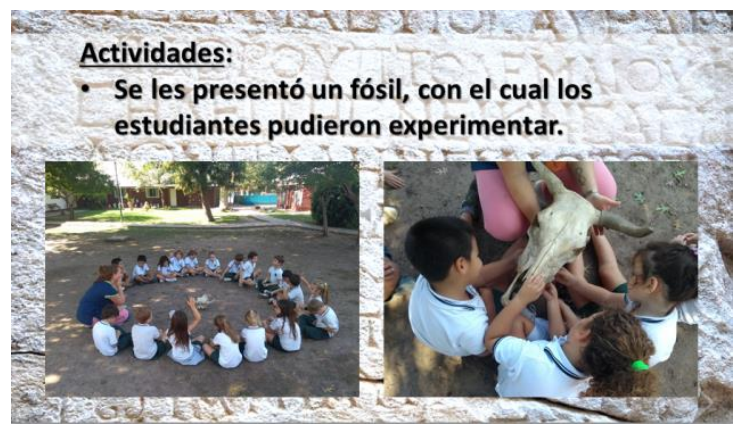

Figura 2

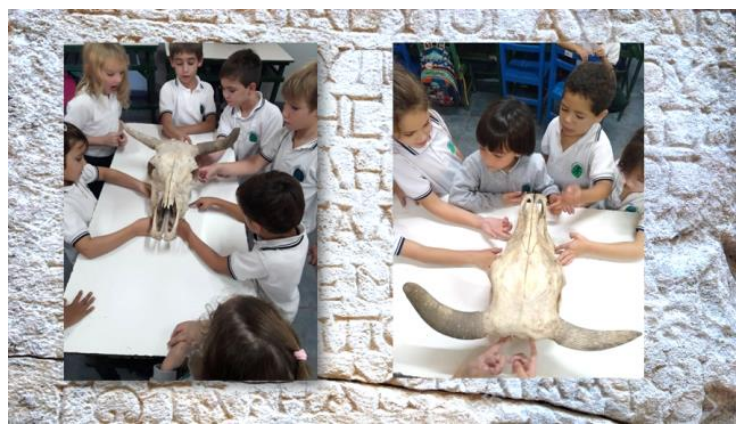

Figura 3 


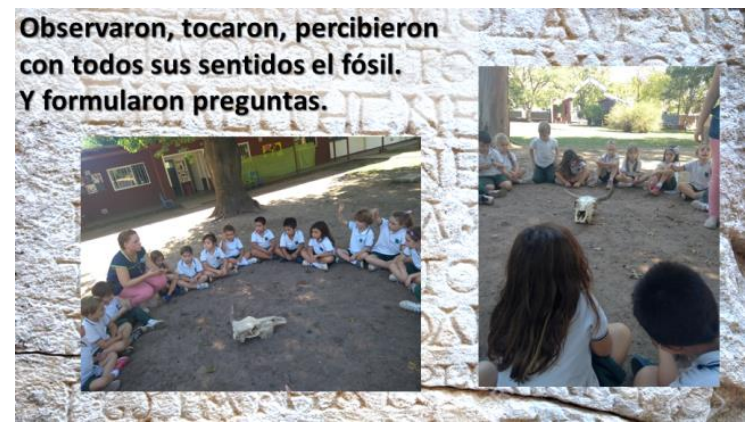

Figura 4

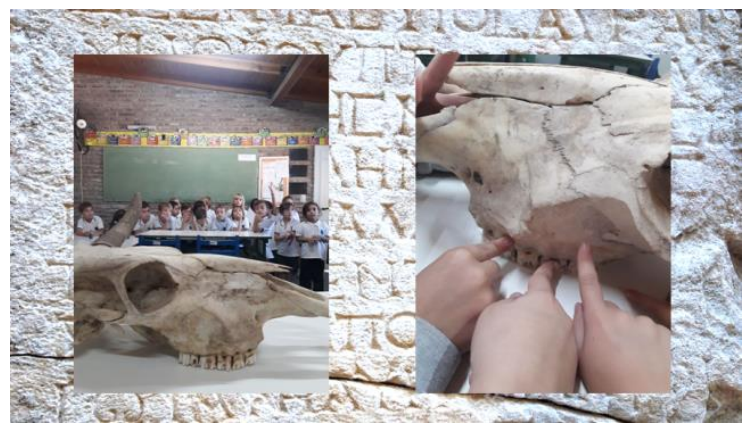

Figura 5

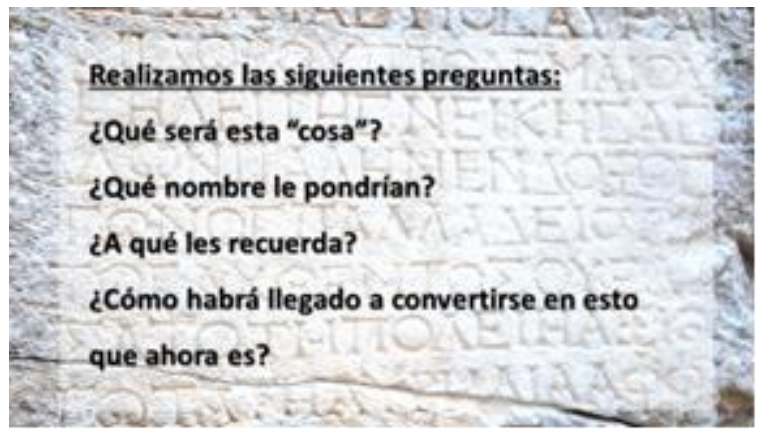

Figura 6 


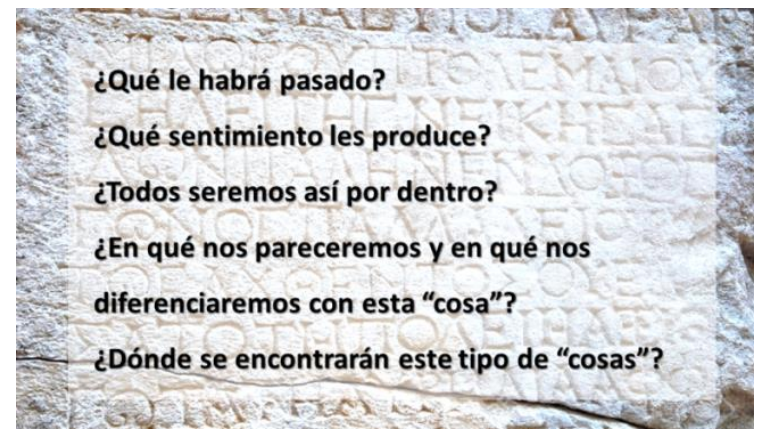

Figura 7

Por otra parte, esta faceta del rizoma Fósil en Ciencias Sociales se centra tanto en la idea práctica de buscar y desenterrar como en la de involucrar el cuerpo y las manos en dicho proceso,y por último, en el concepto de testimonio que comenzamos a explorar de manera introductoria. Con esto, además, seguimos los tres ejes de pasado, presente y futuro que son contenidos conceptuales que

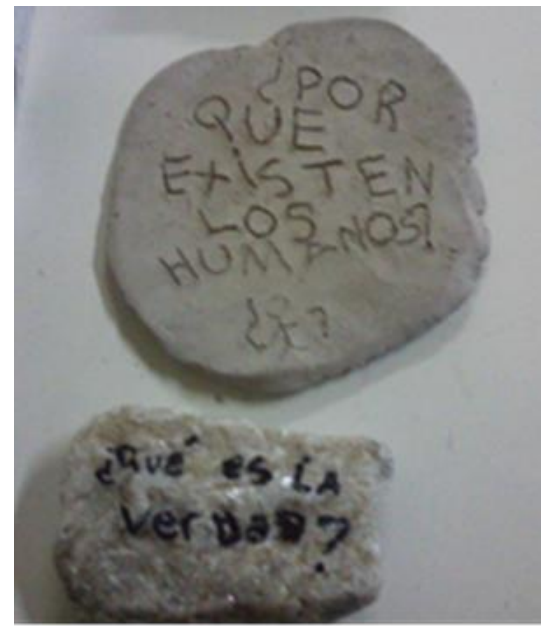
corresponden a los diseños curriculares ministeriales de nuestro país para Primer grado.

A partir de los fósiles que los niños y niñas encuentran y exponen en las clases desarrollamos un camino de preguntas planteadas tanto por los docentes como también por los estudiantes. 

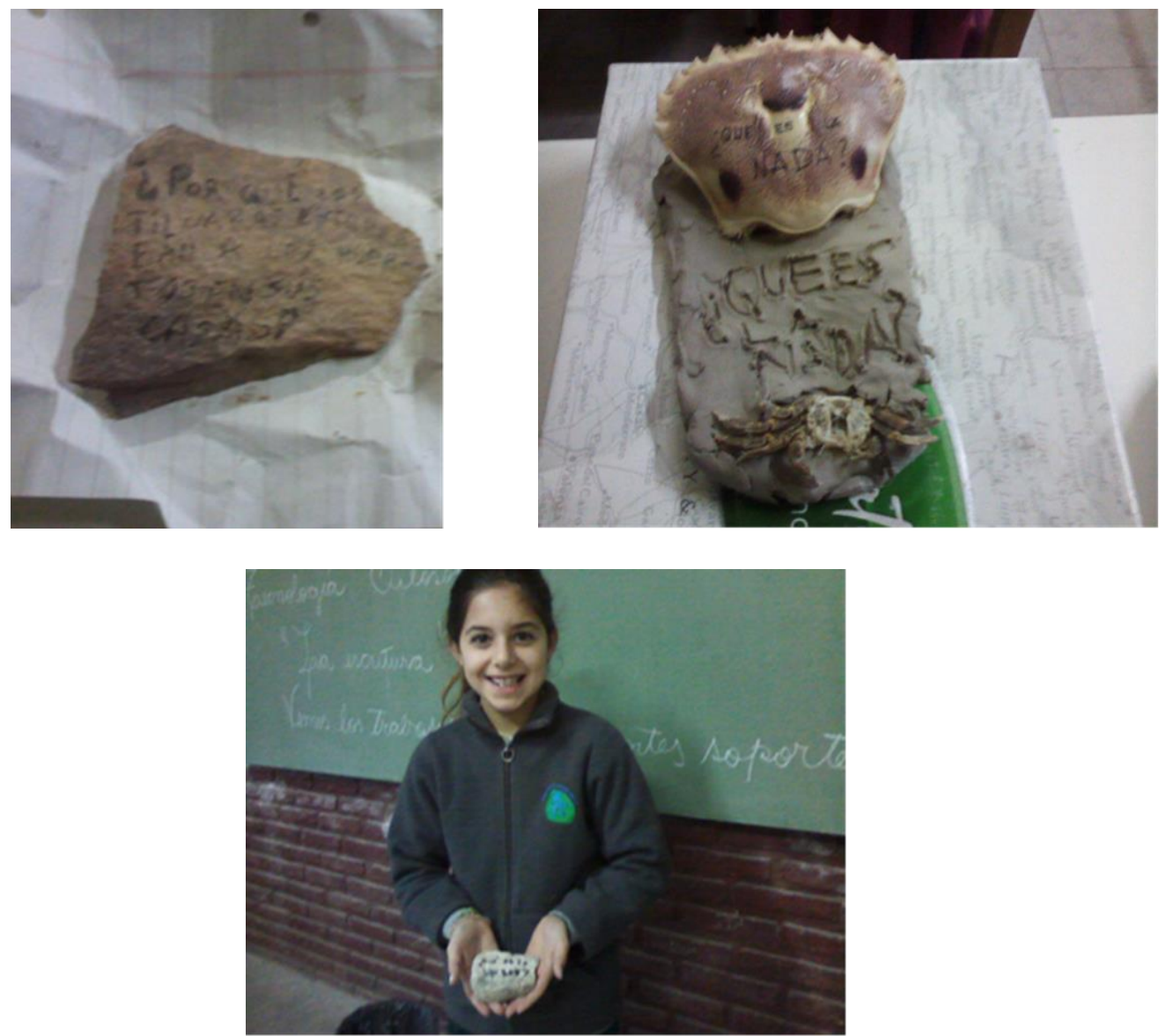

Retomando las preguntas, tomamos como ejemplo las siguientes:

- ¿Qué nos dirá este objeto del mundo en el que habitó?

- ¿Por qué los objetos cambiarán?

- ¿El mundo habrá sido siempre así como lo vivimos ahora?

- ¿En qué tiempo ubicarías este fósil? ¿Pasado, ayer, hoy, el jueves, mañana, anoche, futuro, ahora, presente, ya?

- ¿Podrían agrupar las palabras que significan algo parecido? 
El rizoma se despliega en otras áreas y así se da en su expresión educativa: si seguimos nuestro ejemplo del "rizoma fósil", en el Área de Ciencias Naturales, lo analizamos observando fósiles de diferentes seres vivos y vinculándolos con aquellos que pertenecen al ambiente donde viven: terrestre, aeroterrestre, acuático; según la clasificación instituida en los Diseños Curriculares de Primer grado.

Nos centramos en el

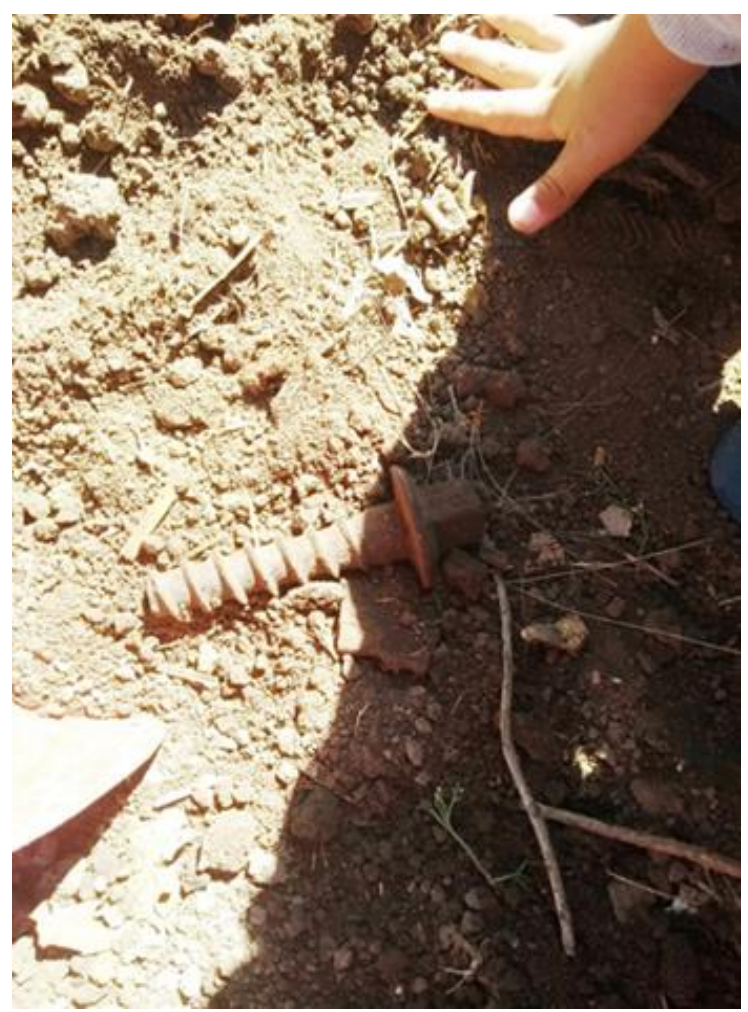
contenido curricular "suelo" a partir de la contraposición, entre seres vivos y seres no vivos en relación a dicho contenido. En este contexto trabajamos la noción de fósil como ser no vivo que habita enterrado en el suelo. ${ }^{18}$

\footnotetext{
${ }^{18}$ En este punto realizamos una articulación con el Espacio de Definición Institucional: Huerta y con el Área Curricular Tecnología. La problemática de la relación entre la búsqueda arqueológica y el trabajo hortícola, la contraposición intervención cultural / espacio natural, la relación entre un elemento no vivo enterrado y un ser vivo que es un producto alimenticio, son algunos de los temas a deconstruir en ambas áreas.
} 

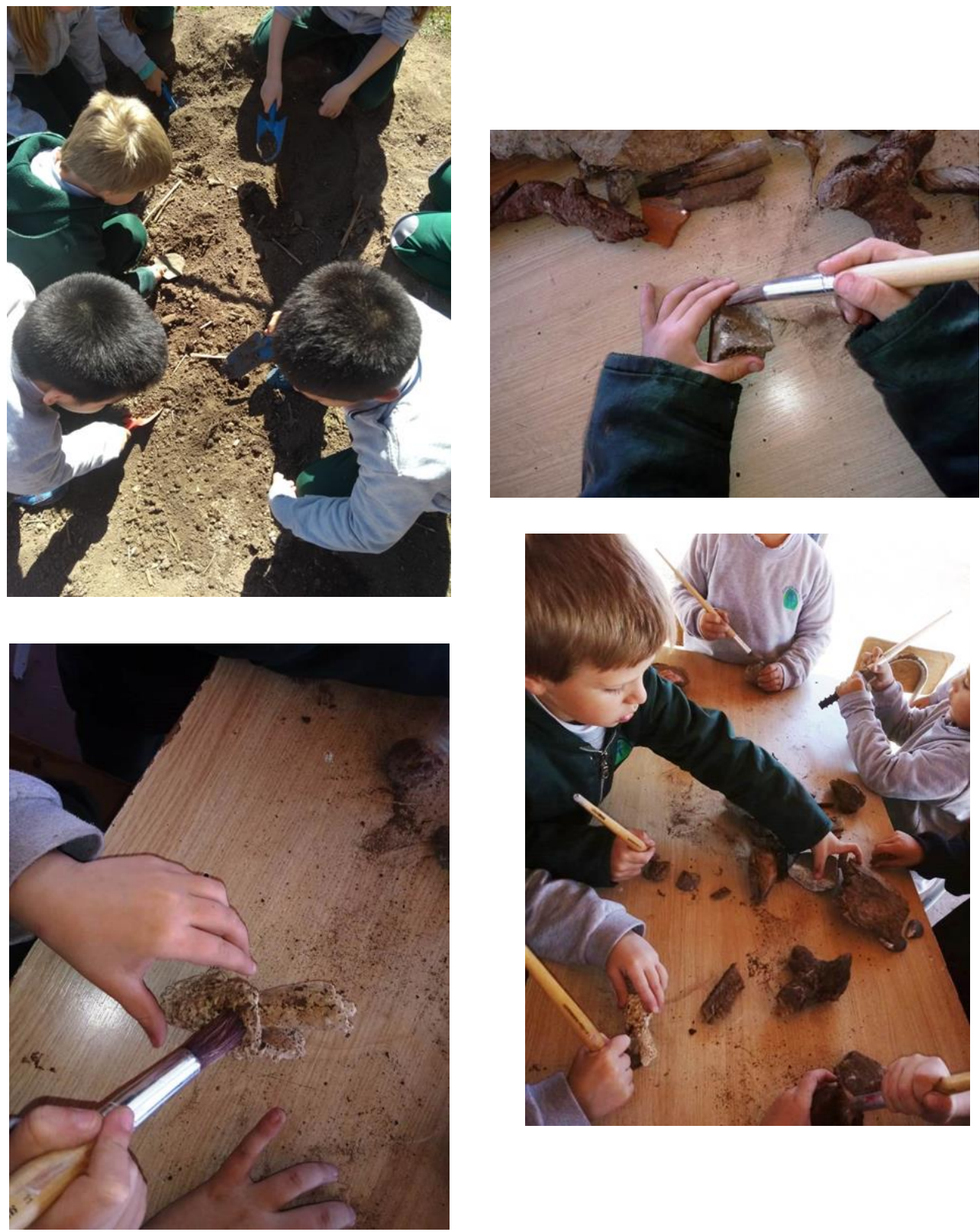

HASER. Revista Internacional de Filosofía Aplicada, no 10, 2019, pp. 37-64 
Luego de realizar esta experiencia y de la observación de los hallazgos compartimos un diálogo basado en preguntas realizadas por los docentes y estudiantes:

- ¿Cómo se imaginan que conviven los seres vivos y no vivos debajo del suelo?

- ¿Las raíces de las plantas son fósiles?

- ¿Un tornillo es un fósil?

- ¿Qué es un fósil?

- ¿Qué pasaría si el suelo fuese transparente?

Asimismo, en Matemática dos de los ejercicios que llevamos a cabo en este rizoma se vinculan, por un lado, con material audiovisual, específicamente un breve cortometraje que muestra esqueletos bailando mientras transcurren las horas:
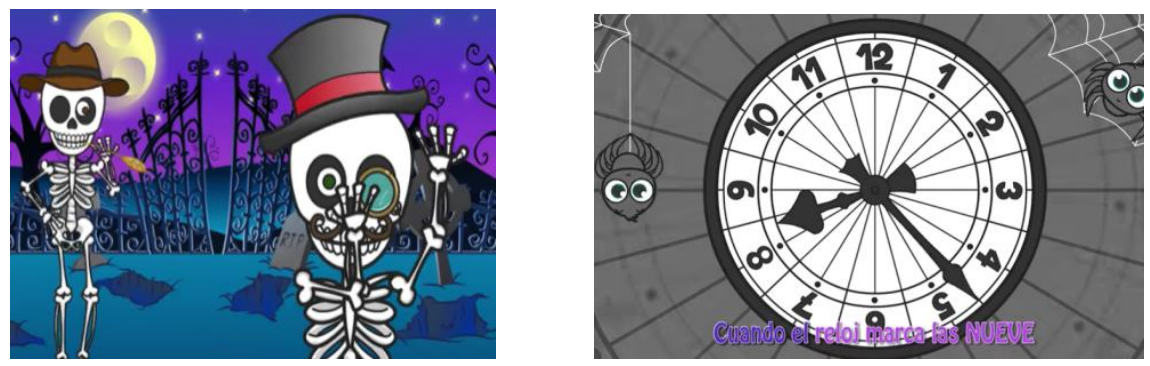

Con ello trabajamos la noción de tiempo como secuenciación de acontecimientos. Y por otro lado, se relacionan con la exposición de fósiles que los estudiantes contarán. Luego se esconderán dichos fósiles y se realizará el siguiente diálogo a partir de preguntas por parte del docente y los estudiantes:

${ }^{19}$ Véase https://www.youtube.com/watch?v=UdB_bFcKb3A 
1. ¿Qué vemos en la historia?

2. ¿Qué serán los números?

3. ¿Dónde estarán los números?

4. ¿Existirán en nuestra mente? ¿En las cosas? ¿En nuestros cuadernos? ¿En esta lista?

5. ¿Podremos formar números con nuestro cuerpo? Experienciemos.

6. Lo que acabamos de realizar con nuestro cuerpo, ¿es el número o la representación del número? ¿Por qué?

7. ¿Si borrásemos todos losnúmeros delmundo, y no pudiésemos formarlos ni con palitos, ni con las manos, ni con películas, con nada, seguiríanexistiendo?

Finalmente cerraramos esta actividad con una danza numérica: los estudiantes realizan movimientos corporales imitando a los números. $^{20}$

El contenido a trabajar en la actividad mencionadaes el conteo de números naturales.En esta oportunidad utilizamos el mismo cortometraje breve para llevar a cabo una actividad en el área de Tecnología ya que en el mismo se destaca el producto tecnológico del reloj. ${ }^{21}$ Además, ligamos estas problemáticas con el espacio

${ }^{20}$ La visibilidad del cuerpo desde la perspectiva según la cual "somos cuerpo" y no pensando la corporeidad como algo ajeno a nosotros mismos o como algo que "tenemos" es crucial en nuestro Método. Por ello, las actividades realizadas intentan involucrar lo humano en su problematicidad integral, mostrando y poniendo en cuestión las dicotomías históricas de alma y cuerpo, mente y cuerpo, etc. Estos temas son abordados también rizomáticamente, en relación, asimismo, con la Educación Sexual Integral.

${ }^{21}$ Asimismo, en el marco del trabajo en Tecnología, continuando la actividad realizada en Matemática nos centraremos los y las docentes en mostrar diferentes tipos de objetos utilizados para medir el tiempo, y también solicitaremos a los estudiantes que los traigan, haciendo hincapié en que investiguen relojes o modos de medir el tiempo que puedan encontrar con sus abuelos u otros familiares o conocidos, que expongan las características de épocas pasadas, sidas. Como también relojes de novedosa y reciente fabricación. De este modo, pondremos en juego las comparaciones, la contextualización y la historización. 
curricular de Ciencias Sociales que, desde otra perspectiva rizomática, piensa el tiempo, como ya señalamos más arriba.

Para concluir con los ejemplos de trabajo rizomático de nuestro Método en torno al fósil nos ocupamos del campo disciplinar de la Construcción Ética y Ciudadana.

Una de las facetas en la que nos detenemos tiene que ver con la idea de aquello y aquellos que existieron y dejaron de existir relacionada al recuerdo familiar, ya que "lo familiar" es un contenido curricular del área indicada. Por otra parte, vincularemos la noción de testimonio introducida en el área de Ciencias Sociales, y de la coexistencia con "lo sido" que nos interpela en esta experiencia de encontrarnos con los fósiles hallados cerca de la cima del volcán Llullaillaco, en el oeste de la provincia de Salta, noroeste de Argentina y que actualmente se encuentran en exhibición en el Museo de Arqueología de Alta Montaña de Salta.

Más allá de hacer la referencia del lugar y el tiempo durante el cual permanecieron enterrados, aproximadamente quinientos años, y con el motivo de tratarse de estudiantes de Primer Grado nos centramos en problematizar los movimientos empáticos y éticos mediante la exposición de las siguientes imágenes y la realización, como siempre conjunta, entre docentes y estudiantes, de una serie de preguntas.

Ellos son los llamados Niños de Llullalaico.

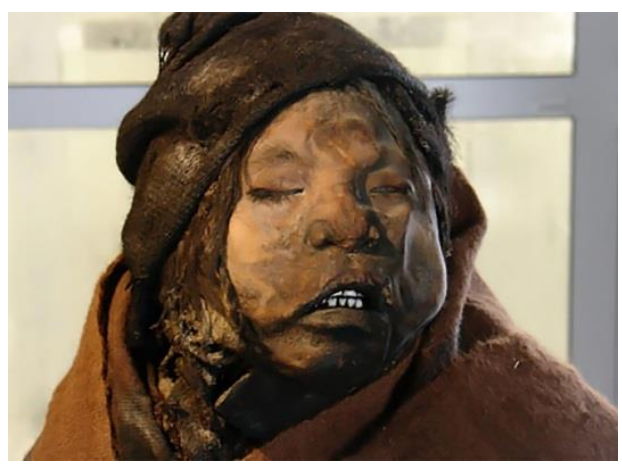



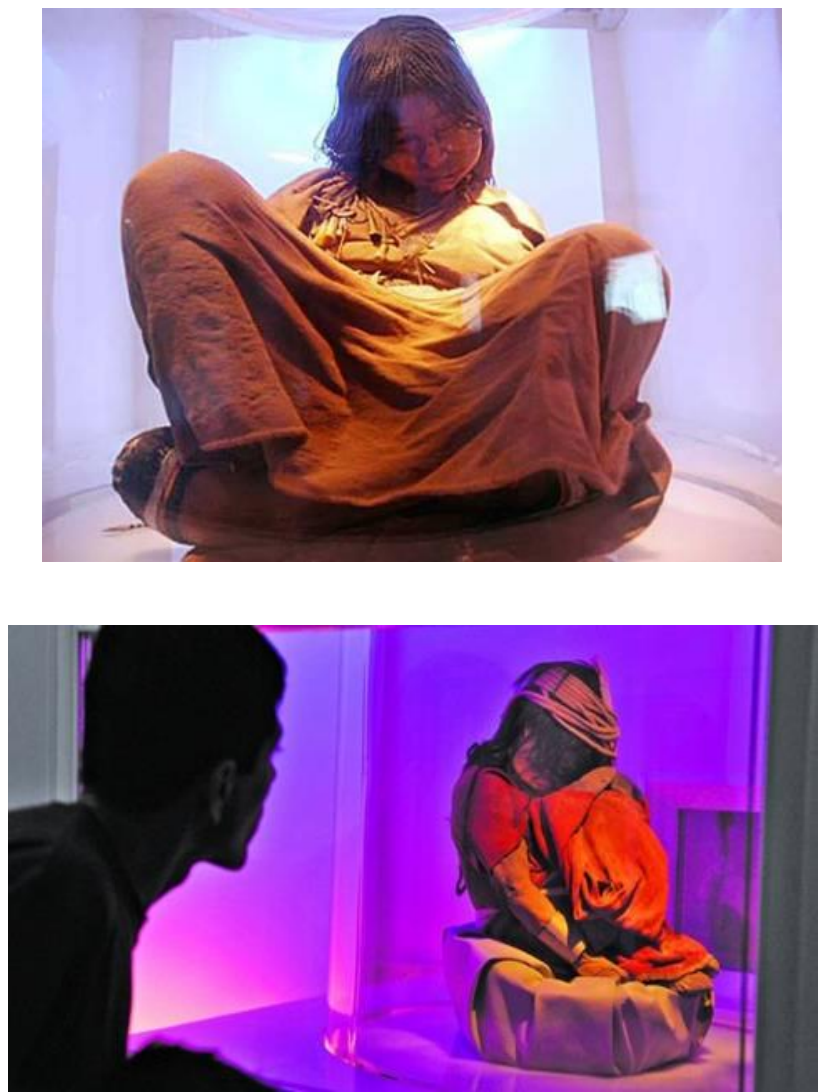

- ¿Qué ven?

- ¿Qué sienten al verlos?

- ¿Por qué creen que ellos estuvieron enterrados allí?

- ¿Por qué creen que ahora ellos están en el museo?

- ¿Quiénes serán?

- ¿Serán amigos, hermanos? ¿Por qué? ¿Qué otra idea se les ocurre?

- ¿Cómo habrán llegado a estar enterrados?

- ¿Qué nos dicen sobre su historia de vida?

- ¿En qué se parecen y en qué se diferencian de nosotros? 
- ¿Son fósiles?

- ¿Seremos nosotros algún día fósiles?

Por otra parte, en las áreas de Especialidades como Plástica, Educación Física, Música, Informática, Inglés, Teatro, Huerta, cada docente se vincula con la planificación del Método a través de su lectura y diálogo con nuestro equipo. De hecho, es un proyecto en el que recién estamos trabajando.Filosofía, además de ser un área especial, atraviesa todo elcurrículo, es decir, todo el discurso rizomáticodel Método Arqueológico Educativo.

\section{En búsqueda de lo evaluable}

De la misma manera estamos en proceso de investigar la práctica tan compleja de la evaluación. En el presente de nuestro trabajo hemos planteado provisoriamente la siguiente división. La evaluación se divide en cuatro instancias simultáneas: 1) La evaluación integrativa, que consiste en un examen que reúne todas las áreas. 2) La evaluación singular por áreas, la más tradicional. 3) La autoevaluación que incluye a) la rememoración por parte de los estudiantes de los rizomas; b) la conversación sobre la calidad de los diálogos -siguiendo la propuesta de Gustavo Santiago ${ }^{22}$ : Diálogo: calidad, ritmo, focalización en un tema, profundidad, interés, complejidad, composición comunitaria. Actitudes: escucha, respeto, atención, solidaridad, participación. Herramientas filosóficas: Utilización. Por otra parte, estamos atentos a los trabajos de José Barrientos en cuanto a su metodología de evaluación, en sus investigaciones recientes, aún no publicadas.

22 Santiago, Gustavo, Filosofía, niños escuela. Trabajar por un encuentro intenso, Paidós, Buenos Aires, 2006. 
Así, Heidegger, Foucault, Barrientos, Deleuze, Derrida, Guattari, Kohan, Agamben, Lipman, López, dibujan un espacio revoltoso de inspiración y de convocatoria, que merece la invención filosófica necesaria para la conversión educativa que buscamos. Apuestas donde la Filosofía se inmerge como práctica infantil, desafío, y aventura arqueológica que arriesga y a la vez, devela nuevos trayectosrememorantespara experimentar y edificar el habitar educativo escolar.

Privar a los niños y niñas de conocer los distintos ángulos de las condiciones de producción de los saberes y la implicancia del tránsito de la propia experiencia es detener la potencia infantil, deseducar, anular las infinitas posibilidades, anular lo infinito de lo posible.

\section{Bibliografía}

Agamben, Giorgio: Infancia e historia. Destrucción de la experiencia y origen de la historia, Adriana Hidalgo, Buenos Aires,2007.

Barrientos, José, "La experiencialidad como respuesta a la tendencia analítica de la filosofía para niños" en Childhood\&phylosophy, V. 12, n. 25, Río de Janeiro, 2016.

Cázares Aponte, Leslie: Imbricar Filosofía para Niños y Jóvenes en los centros escolares. Una perspectiva de inclusión escolar para toda la comunidad educativa, Octaedro Recursos, Barcelona, 2014 Deleuze, Gilles; Guattari, Félix, "Rizoma", en Mil Mesetas. Capitalismo y esquizofrenia, Pre-textos, Valencia,2004.

Foucault, Michel: La arqueología del saber, Siglo XXI, Buenos Aires, 2002.

Hermenéutica del sujeto. Entrevista publicada en la Revista Concordia, No 6, Altamira, La Plata: 1984.

La hermenéutica del sujeto, FCE, Buenos Aires,2006. 
Gagnebin, Jeanne Marie: "Infância e pensamento", en Sete Aulas sobre Linguagem, Memória e História. Imago, Río de Janeiro, 2006.

Cragnolini, Mónica: "Derrida: deconstrucción y pensar en las "fisuras", en Ciclo "El pensamiento francés contemporáneo, su impronta en el siglo, Conferencia en la Alianza Francesa, Buenos Aires, 30 de setiembre de 1999.

Heidegger, Martín: "La cosa", en Conferencias y artículos, Ediciones del Serbal, Barcelona, 1994.

¿Qué es eso de filosofía?, Sur, Buenos Aires, 1960.

Kohan, Walter - Waksman, Vera: Filosofía con niños. Aportes para el trabajo en clase, Novedades educativas. Buenos Aires, 2005.

El maestro inventor. Simón Rodríguez, Ediciones del Solar, Caracas, 2016.

Kohan, Walter - Rodriguez, Allan, Berle, Simone: Filosofía e Filosofía e Educaçãoemerrância: inventar escola, infâncias do pensar, NEFI, Río de Janeiro, 2018,pp. 9-17.

Lipman, Matthew - Sharp, Ann - Oscayán, Frederick: La filosofía en el aula, Ediciones de la Torre, Madrid, 1992.

López, Maximiliano: Filosofía con niños y jóvenes. La comunidad de indagación a partir de los conceptos de acontecimiento y experiencia trágica, Noveduc, Buenos Aires, 2008.

Santiago, Gustavo, Filosofía, niños escuela. Trabajar por un encuentro intenso, Paidós, Buenos Aires, 2006. 